\title{
bases
}

\section{para establecer un control secuencial de las resistencias de un hormigón en obra}

\author{
JOSE MANUEL LOPEZ SAIZ, \\ Ingeniero de Caminos, Canales y Puertos (A.M.A.S.C.E.)
}

$462-1$

\section{sinopsis}

El presente artículo intenta disponer las bases para un posible sistema de control de hormigones por lotes, de forma que a partir de él se pueda establecer un conjunto homogéneo de normas que permitan un sistema de control eficiente y estandarizado.

Al pretender controlar hormigones en obra, siguiendo los criterios estadísticos establecidos en las últimas normas de aplicación (HA 61-M.O.P.1968), el único sistema a seguir es confeccionar probetas de las amasadas vertidas en obra, representar la resistencia de esa amasada por valor obtenido de esas probetas, y considerar esto como una muestra perteneciente a una población constituida por todo el hormigón de ese tipo puesto en obra, y entonces, a partir de las muestras obtenidas, estimar los parámetros $\mu$ y $\sigma$ de dicha población.

Debido a que el control se efectúa mediante un valor, función de $\mu$ y $\sigma$, el cual caracteriza a la población frente a su adecuación a las necesidades del proyecto, es también posible realizar estimaciones directas de este parámetro $\left(R_{k}=\mu-\alpha \sigma\right)$ sin necesidad de efectuar cálculos previos de $\mu$ y $\sigma$.

Las condiciones que debe reunir un estimador no son tratadas aquí, pero sí conviene aclarar que todo estimador de la resistencia característica tiene a su vez una función de distribución cuya media coincidirá con $R_{k}$ (caso de ser centrado) y cuya desviación típica será función de $\sigma$. Esto quiere decir que si nosotros calculamos mediante un cierto estimador el valor de $R_{k}$, a partir de un muestreo el valor obtenido $E_{k}$ será mayor o menor que $R_{k}$, pudiendo aparecer desviaciones mayores o menores según la desviación típica del estimador.

Ocurre, pues, que al estimar la resistencia característica de un hormigón se corre el riesgo de suponerlo menor que el real un cierto número de veces (riesgo del vendedor) y de suponerlo mayor que el real en otros casos (riesgo del comprador).

Sin embargo, no parece lógico que el riesgo del comprador y del vendedor sean iguales; pues si bien es cierto que si un hormigón tiene una 
resistencia característica igual o mayor que la del cálculo es totalmente aceptable, no es menos cierto que si la tiene menor vaya a ser automáticamente rechazado.

Evidentemente, el caso de los hormigones no puede tratarse como el de un lote de piezas en el cual la aceptación o rechazo son automáticos, porque en estos casos el riesgo del vendedor se suele hacer pequeño, ya que el riesgo del comprador siempre es menos grave (puede haber un número de piezas defectuosas mayor que el estipulado, pero siempre quedan piezas buenas y aprovechables).

Para poder, entonces, establecer un criterio de aceptación y rechazo automático sería necesario definir previamente una posición de riesgo de vendedor (fabricante) y comprador (proyecto), posición muy difícil dado que la aceptación de hormigones con resistencias por debajo de la característica sólo puede hacerse tomando precauciones adicionales, estudio más detallado, pruebas de carga, obtención de testigos, etc., los cuales exigen un gasto y, sobre todo, crean la necesidad de estimar de algún modo esta resistencia característica.

Resulta, por tanto, que el control debe llevarse a cabo a través de estimaciones de la resistencia característica del hormigón, estimaciones que, como ya hemos dicho, tienen desviaciones con relación al verdadero valor; de lo cual se deduce que un sistema de control interesante sea el control por lotes.

Pretendemos aquí, únicamente, dar unas ideas de cómo se puede llegar a establecer un control de este tipo, sin proponer valores ni coeficientes que deben ser, en todo caso, discutidos con gran cuidado y en comisiones en las que intervengan siempre representantes tanto del comprador como del vendedor.

Para realizar un control de este tipo es necesario:

1. Definir el tamaño del lote.

2. Definir el número de series mediante las que se va a controlar este lote. Debido a que el mayor o menor número de series empleado en el control de un lote define la precisión obtenida en ese control, se deben establecer tres niveles en el mismo, control reducido, normal y riguroso, correspondiendo a cada uno de ellos un número de series mayor que el anterior.

3. Definir el número de probetas que compondrán cada serie. Cada serie se caracterizará por la media de las resistencias obtenidas en cada una de las probetas.

Con el fin de evitar, en lo posible, que los errores de ensayo y la variabilidad del mismo se añadan a la variabilidad del hormigón fabricado, es necesario que cada serie se componga al menos de tres probetas para poder obtener un estimador suficientemente preciso de la media del ensayo.

4. Establecer el estimador a emplear: Este debe ser conocido en cuanto a su media y desviación típica y es muy conveniente conocer también su función de distribución, ya sea de forma analítica, ya sea obtenida en forma numérica por un proceso de integración aproximado, ya sea mediante un proceso de simulación. En caso de no conocer la función de distribución se puede em- 
plear, para obtener aproximaciones de los datos que indicamos a continuación y que son necesarios para este tipo de control, el teorema de Tchevicheff, si bien éste nos dará aproximaciones muy groseras que exigirán un gran cuidado en el momento de plantear el control.

A partir de los datos anteriores se calcularán:

a) Probabilidad de aceptación y rechazo de un lote en función de la relación entre resistencia característica real del lote y resistencia característica exigida ( $R_{c}$ real $/ R_{c}$ exigida).

b) Probabilidad de cada uno de los valores que puede tomar el estimador en función del coeficiente $\left(E_{c} / R_{c}\right.$ real), es decir, relación entre el valor estimado y el real de la resistencia característica.

Por supuesto que los valores estimados en $a$ ) y $b$ ) varían con el coeficiente de variación del hòrmigón y con el tamaño de la muestra empleada.

5. Establecer los límites de control en función de los datos obtenidos anteriormente.

5.1. Estos límites se establecerán en:

a) Valor que tiene muy pocas probabilidades $(0,02)$ de ser superado si $R_{c}$ lote $=R_{c}$ exigida, y le llamaremos $L_{M}$.

b) Valor que tiene pocas probabilidades $(0,05)$ de ser superado en el mismo caso $\left(L_{m}\right)$.

c) Valor por debajo del cual hay pocas probabilidades $(0,05)$ de obtener estimaciones de la resistencia característica en las mismas condiciones de los casos anteriores $\left(L_{i}\right)$.

d) Valor que tiene muy pocas probabilidades $(0,02)$ de que haya estimaciones por debajo del mismo en las condiciones anteriores $\left(L_{I}\right)$.

6. Se comienza el control a un nivel intermedio y se efectúa la estimación de la resistencia característica del lote; entonces puede ocurrir que el valor estimado:

a) Esté por encima de $L_{M}$.

Se puede optar por:

a.1) pasar a un control reducido;

a.2) modificar las dosificaciones para ajustarse a las exigencias del proyecto.

b) Esté entre $L_{M}$ y $L_{m}$ : Se pasa a un nivel más reducido de control, si lo hay; en caso de estar en el nivel de control más reducido actuar como en a).

c) Se encuentre entre $L_{m}$ y $L_{i}$ : El hormigón se acepta y se aplica al mismo una bonificación o una multa proporcional al valor de $E$ estimada/ $R$ exigida. Evidentemente se debe aplicar lo mismo un premio al obtener estimaciones superiores a $R_{c}$ que una multa al obtenerlas menores, ya que el estimador puede darnos valores por encima o por debajo del valor real; debido a ello, si solamente se penalizan los valores por debajo de $R_{c}$ sin premiar los otros tendríamos casos como éste: 
Supongamos un estimador centrado y simétrico: En este caso, si el hormigón tiene $R_{c}$ hormigón $=R_{c}$ exigida, al estimar el valor de $R_{c}$ hormigón en cada lote obtendremos (teóricamente) un $50 \%$ de valores por debajo de $R_{c}$ y un $50 \%$ por encima. Si penalizamos las que son menores que $R_{c}$ habremos llegado al absurdo de penalizar la mitad de los lotes que forman un hormigón que cumple las condiciones exigidas. Si, por el contrario, premiamos el hormigón con resistencias superiores en función de la relación $E_{c} / R_{c}$, debido a que $\bar{E}_{c}=R_{c}$ resultará al final que premios y multas se compensan.

d) Se encuentre entre $L_{i}$ y $L_{I}$ : Se pasa a un control más estricto al tiempo que se penaliza el hormigón como en el caso anterior, salvo que estemos en el nivel de control mắs riguroso, en cuyo caso se actuará como en $e$ ).

e) Es inferior a $L_{I}$ : El hormigón debe ser modificado en su dosificación para que cumpla las exigencias del proyecto, y estudiada detenidamente la aceptación o rechazo del mismo, según las exigencias, de una resistencia mínima.

7. Al terminar el control se comprobará el montante de premios y multas. Si en conjunto resulta que el hormigón ha sido bonificado se anula dicha bonificación, ya que a nadie interesa premiar hormigones por encima de las necesidades de proyecto. Si en conjunto resulta una multa para el suministrador se deberá aplicar la misma de acuerdo con una cierta ley que, a mi entender, debe ser parabólica (Cfr. «Proposición de Pliego de Condiciones y precios de contratación para hormigón suministrado en obra», INFORMES DE LA CONSTRUCCIÓN, número 213).

Cuando el número de series, conjunto de diferentes lotes, es suficientemente grande (más de 30) se puede calcular, con suficiente precisión, la resistencia característica mediante el estimador:

$$
M=\bar{X}-K \cdot S\left\{\begin{array}{l}
K=1.645 \text { para } n>30, \\
K=f(n) \text { para } n<30,
\end{array}\right.
$$

pues en este caso para $V=0,1$ el $99,7 \%$ de los resultados difiere del valor real en menos de un $5 \%$ y para $V=0,15$ el $99,7 \%$ de los valores obtenidos difiere del valor real en menos de un $8 \%$ $\mathrm{y}$, en las mismas condiciones, un $95,5 \%$ difiere en menos del $3 \%(V=0,1)$ y para $V=0,15$ la misma proporción difiere en menos de un $5,4 \%$.

Es muy importante tener en cuenta que en todo lo indicado anteriormente se ha supuesto que el hormigón que estamos controlando pertenece a una única población.

Se puede efectuar en cada lote una comprobación de que, efectivamente, este último lote pertenece a la misma población que el conjunto formado por los lotes anteriores.

Para ello obtenemos la media $R_{m l}$ y la varianza $S_{l}{ }^{2}$ del último lote, compuesto de $n_{l}$ series, y la media $R_{m a}$ y la varianza $S_{a}^{2}$ de los $n_{a}$ resultados anteriores. A partir de estos valores se calcula:

$$
t=\left(R_{m a}-R_{m l}\right) \sqrt{\frac{n_{l} \cdot n_{a}\left(n_{l}+n_{a}-2\right)}{\left(n_{l}+n_{a}\right)\left(n_{l} \cdot S_{l}^{2}+n_{a} \cdot S_{a}^{2}\right)}}
$$


resultando que $t$ se distribuye según una función $t$ de Student de $\left(n_{i}+n_{a}-2\right)$ grado de libertad, pudiendo comprobarse entonces si la probabilidad de obtener tal valor de $t$ es grande o pequeña; si la probabilidad de obtener ese valor de $t$ es menor que un cierto valor establecido de antemano diremos que este último lote no pertenece a la población anterior. En ese caso se deberá establecer nuevas medidas (comienzo del control, revisión de las dosificaciones, etc.).

Todo esto puede parecer complicado, pero su programación en un ordenador es muy sencilla, por lo cual, y dado que este tipo de control exige ya una especialización, debe ser éste el camino a seguir para llevarlo a la práctica.

Por último, hay que hacer constar una de las mayores dificultades de este sistema de control por lotes, y es el de que las decisiones han de ser tomadas, lo más pronto, 28 días después de ejecutadas las últimas probetas del lote; ello quiere decir que si es necesario cambiar el nivel de control, sobre todo si es a uno más riguroso, quizá no sea posible hacerlo ya en el lote siguiente, que, si la obra es rápida, quizá se haya terminado a su vez.

Por todo ello, este sistema de control, que puede ser muy interesante, sólo se puede potenciar al máximo si al tiempo se halla un sistema de obtención de las resistencias de control a los 2 ó 3 días, como máximo, de la ejecución de las probetas.

\section{résumé}

Bases pour établir un contrôle séquentiel des résistances d'un béton sur chantier José Manuel López Saiz, ingénieurs des Ponts et Chaussées (A.M.A.S.C.E.)

Dans cet article, l'auteur tente de disposer les bases pour un possíble système de contrôle de bétons par lots, de façon qu'à partir de lui on puisse établir un ensemble homogène de normes permettant d'assurer un système de contrale efficlent et standardisé.

\section{summary}

Basis for a Sequence Control of Concrete Strenth at the Site

José Manuel López Saiz, Civil engineer (A.M.A.S.C.E.)

This paper seeks to establish the bases for a possible control of concrete by lots, so that a homogeneous set of specifications can be derived, leading to an efficient and standardised system of quality and performance control.

\section{zusammenfassung}

Grundlagen für eine Sequenzkontrolle der Widerstände in einem Baubeton

José Manuel López Saiz, Tlefbauingenieur (A.M.A.S.C.E.)

Mit diesem Artikel wird der Versuch unternommen, die Grundlagen eines möglichen Kontrollsystems für Baulosbeton festzulegen, von denen aus ein homogener Normenkomplex erstellt werden könnte, um so eln wirksames Standardkontrollsystem zu ermöglichen. 